\title{
Long-term safety of long-acting octreotide in patients with diabetic retinopathy: results of pooled data from 2 randomized, double- blind, placebo-controlled phase 3 studies
}

\author{
Rosario Pivonello ${ }^{1}$ Giovanna Muscogiuri ${ }^{1}$ - Geoffrey Holder ${ }^{2}$ - Michaela Paul ${ }^{2}$. \\ Severine Sarp ${ }^{2}$ - Anastasia Lesogor ${ }^{2} \cdot$ Pierre Jordaan $^{2} \cdot$ Johannes Eisinger $^{2}$. \\ Annamaria Colao ${ }^{1}$
}

Received: 23 August 2017 / Accepted: 30 September 2017 / Published online: 7 November 2017

(C) The Author(s) 2017. This article is an open access publication

\begin{abstract}
Purpose Octreotide (OCT) has been successfully used for treatment of acromegaly and neuroendocrine tumors for more than 30 years. However, long-term safety of OCT has not been documented in placebo-controlled setting. This present analysis pooled safety data from two similarlydesigned, randomized, and placebo-controlled studies to evaluate long-term safety of long-acting OCT $(20,30 \mathrm{mg})$; targeted post-hoc analyzes focused on cardiac, hepatic, and renal safety.

Methods Two studies (NCT00131144, NCT001308450) were conducted in patients with diabetic retinopathy $($ OCT $20=191$, OCT $30=348$, placebo $=347)$. In this analysis, patients were stratified based on baseline glomerular filtration rate. Hepatic, cardiac, and renal adverse events (AEs) were identified by standardized MedDRA queries.

Results Median duration of exposure was $>3.5$ years. Most common AEs reported with OCT were diarrhea, cholelithiasis, hypoglycemia, nasopharyngitis, and hypertension. Incidence of cardiac events (QT prolongation and arrhythmia) with OCT20 and OCT30 were comparable to placebo (OCT20, RR = 1.11 [95\% CI, 0.61-2.03]; OCT30, RR = 1.09 [95\% CI, 0.70-1.68]). For ECG findings, changes in QTcF were similar in treatment groups, and outliers did not exceed $480 \mathrm{~ms}$. Incidence of cardiac ischemia was lower with OCT than placebo $(\mathrm{OCT} 20=12.6 \%$, ОCT $30=$
\end{abstract}

Rosario Pivonello

rosario.pivonello@unina.it

1 Dipartimento di Medicina Clinica e Chirurgia, Sezione di Endocrinologia, Università Federico II di Napoli, Naples, Italy

2 Novartis AG, Basel, Switzerland
$10.6 \%$, placebo $=15.3 \%)$. Incidence of liver-related AEs was higher with ОСТ30 than placebo $(\mathrm{RR}=2.04$ [95\% CI, 1.28-3.26]); incidences were comparable with OCT20 and placebo $(\mathrm{RR}=1.50$ [95\% CI, 0.69-3.25]). Overall incidences of renal AEs were comparable between treatment groups $(\mathrm{OCT} 20=5.8 \%$; ОCT $30=6.3 \%$; placebo $=7.2 \%)$. Drug-related SAEs were reported more frequently with OCT $($ OCT2 $=7.9 \%$; OCT30 $=10.1 \%$; placebo $=3.5 \%)$; predominantly gallbladder-related, GI-related, and hypoglycemia.

Conclusions The results from these long-term placebocontrolled studies confirm the established safety profile of long-acting OCT, in particular low risk of cardiac, hepatic and renal toxicity in a high-risk population.

Keywords Diabetic retinopathy $\cdot$ Octreotide $\cdot$ Long-term safety $\cdot$ Renal function $\cdot$ Hepatic function $\cdot$ Cardiac function

\section{Introduction}

Octreotide (SMS 201-995, octreotide acetate, Sandostatin ${ }^{\circledR}$, Novartis) is a somatostatin analogue (SSA) with a therapeutic use in pathophysiological states associated with excessive hormone production and secretion [1-4].

With extensive clinical and real-world use over the past 30 years, octreotide is considered to have a favorable benefit-risk profile in acromegaly and neuroendocrine tumors [5-8]. The majority of adverse events (AEs) are due to the octreotide-induced imbalance of hormonal pathways, most of which occur in the gastrointestinal (GI) tract manifesting commonly in diarrhea, flatulence, abdominal pain and nausea. Other AEs associated with octreotide include 
hyperglycemia and abnormalities of cardiac rhythm. Hyperglycemia is a common reaction which may be caused by inhibition of insulin secretion. Patients with acromegaly often exhibit underlying insulin resistance because of their excess of growth hormone, and the degree of impairment in glucose homeostasis potentially brought about by SSA treatment is debated in the literature [7, 9, 10, 11]. Abnormalities of cardiac rhythm are clinically relevant in acromegaly. Octreotide has been shown to decrease the heart rate and prolong the QT interval, an important risk factor for ventricular arrhythmias. Although this may be perceived as a treatment risk, there is, however, some evidence that octreotide may have a beneficial cardiac effect in patients with acromegaly with a reduction, and in some cases, normalization of QT intervals [12] and with improvement of cardiac performance and hypertension [13, 14].

Long-term safety of octreotide has been the subject of several published studies and reviews; however, findings associated with octreotide treatment in placebo-controlled studies exceeding 30 months have not been documented. Therefore, the results of two randomized double-blind, placebo-controlled, parallel-group studies present a unique opportunity to assess the safety of octreotide in a vulnerable patient population (diabetic patients with microvascular disease) with a long-term follow-up period over 2 years.

Octreotide is not currently approved for the treatment of diabetes mellitus or diabetic retinopathy; however, patients with diabetes comprise some of the populations with approved conditions. The results from the studies presented here provide critical insight into the safety and tolerability of long-term octreotide use in a population with a high cardiovascular risk.

\section{Methods}

\section{Description of the pooled trials}

Two randomized, double-blind, placebo-controlled studies were conducted between 1999 and 2006 in patients with diabetic retinopathy with the primary objective of determining whether long-acting octreotide, administered at 20 or $30 \mathrm{mg}$ every 4 weeks, delayed the time to progression of diabetic retinopathy according to defined endpoints on the Early Treatment Diabetic Retinopathy Study final retinopathy severity scale (study 1: CSMS995H0802 [NCT00131144] and study 2: CSMS995H0804 [NCT00130845]). This present analysis pooled the safety data of these two studies to evaluate the overall long-term safety and also in the sub-populations defined by the baseline level of renal function. In addition, the targeted post-hoc analyses also focused on cardiac, hepatic, and renal safety.
Patient population and study design were similar for both studies that differed only in the recruitment region and number of investigational treatment arms (study 1: Europe, octreotide $20 \mathrm{mg}$ [OCT20] and octreotide $30 \mathrm{mg}$ [OCT30]; study 2: North America and South America, OCT30). The studies enrolled male and female patients with type 1 diabetes or type 2 diabetes, irrespective of insulin dependence, between 18 and 70 years with an HbA1c $<13.0 \%$. Patients had moderately severe or severe non-proliferative diabetic retinopathy or low risk proliferative diabetic retinopathy. The main exclusion criteria were a history of brittle diabetes or hypoglycemia unawareness, symptomatic gallstones without a cholecystectomy, a positive pregnancy test, or prior treatment with octreotide or another SSA. Overall, 898 patients were randomized, 585 in study $1(N=195$ on OCT20, $N=197$ on OCT30, and $N=193$ on placebo) and 313 patients in study $2(N=155$ on OCT30, and $N=158$ on placebo).

\section{Compliance with ethical standards}

Study 1 and study 2 were approved by the institutional review boards of the respective institutions prior to patient enrollment. All procedures performed in study 1 and study 2 involving human participants were in accordance with the ethical standards of the institutional and/or national research committee and with the 1964 Helsinki declaration and its later amendments. Informed consent was obtained from all individual participants included in the study.

\section{Safety data}

All analyses were based on the safety analyzable population (SAF), defined as patients who received at least one dose of trial medication and for whom one or more post-baseline safety assessments were obtained. The safety population for study 1 consisted of 578 patients $(N=191$ on OCT20, $N=$ 196 on OCT30, and $N=191$ on placebo) and for study 2 consisted of 308 patients $(N=152$ on OCT30, and $N=156$ on placebo). The safety assessments included laboratory data, electrocardiogram (ECG), vital signs, and gallbladder ultrasound. Single 12-lead ECGs were collected at baseline and at the end of treatment. During the study, ECGs were performed if they were clinically indicated by the patient's symptomatology (as per investigator opinion). All ECGs were assessed centrally by an ECG core laboratory retrospectively for central blinded assessment. For this present analysis, data from the two studies were pooled. Adverse events of special interest (hepatic, cardiac, and renal events) were identified by standardized MedDRA queries (SMQs) based on MedDRA version 18.0, and multiple AEs within the same SMQ were counted only once per patient. 


\section{Sub-populations (based on baseline renal status)}

The diabetes population under study was an opportunity to compare and analyze safety data in patients with impaired as well as normal renal function. Accordingly, analyses were presented in the overall SAF population and by subpopulations defined by renal function at baseline, either as normal or mildly impaired renal function (baseline estimated glomerular filtration rate (eGFR) $\geq 60 \mathrm{~mL} / \mathrm{min} / 1.73 \mathrm{~m}^{2}$ ), and for impaired renal function (baseline eGFR $<60 \mathrm{~mL} /$ $\min / 1.73 \mathrm{~m}^{2}$ ) (Table 1). Renal function was assessed by the abbreviated modification of diet in renal disease eGFR formula [15].

\section{Statistical analysis}

Baseline values were defined as the last non-missing value prior to the first dose of study treatment. Adverse event summaries include treatment-emergent AEs, occurring up to and including 90 days after the date of last study medication. For the AEs of special interest, relative risk (RR, risk ratio of incidence on octreotide treatment vs. placebo) estimates and 95\% confidence intervals (CIs) were calculated using Mantel-Haenszel method and adjusted for study as stratification factor. Pairwise comparisons of OCT30 vs. placebo and OCT20 vs. placebo for each SMQ were performed overall and in the sub-population based on baseline renal status. For the ECG analysis, the ECG recorded at the end of study visit (EOS) was considered as the last ontreatment ECG. The mean, central tendency change for the ECG parameters from baseline to EOS, as well as the number and percentages of ECG events of interest (categorical changes) were summarized.

Table 1 Baseline eGFR $\left(\mathrm{mL} / \mathrm{min} / 1.73 \mathrm{~m}^{2}\right)$ by treatment group and sub-population

\begin{tabular}{|c|c|c|c|c|}
\hline \multirow[t]{2}{*}{ Population } & \multicolumn{2}{|c|}{ Long-acting octreotide } & \multirow[t]{2}{*}{ Placebo } & \multirow[t]{2}{*}{ Total } \\
\hline & $30(\mathrm{mg})$ & $20(\mathrm{mg})$ & & \\
\hline \multicolumn{5}{|c|}{ Baseline eGFR $\geq 60 \mathrm{~mL} / \mathrm{min} / 1.73 \mathrm{~m}^{2}$} \\
\hline$N$ & 237 & 133 & 238 & 608 \\
\hline Mean (SD) & $74.8(9.43)$ & $72.8(9.37)$ & $73.2(8.52)$ & $73.7(9.09)$ \\
\hline Median & 74.3 & 70.9 & 72.7 & 73.1 \\
\hline Min-max & $60-104$ & $60-102$ & $60-98$ & $60-104$ \\
\hline \multicolumn{5}{|c|}{ Baseline eGFR $<60 \mathrm{~mL} / \mathrm{min} / 1.73 \mathrm{~m}^{2}$} \\
\hline$N$ & 110 & 58 & 108 & 276 \\
\hline Mean (SD) & $49.2(8.53)$ & $48.9(10.16)$ & $49.1(9.29)$ & $49.1(9.16)$ \\
\hline Median & 50.9 & 52.1 & 52.4 & 51.7 \\
\hline Min-max & $18-59$ & $21-60$ & $12-60$ & $12-60$ \\
\hline \multicolumn{5}{|l|}{ All patients } \\
\hline$N$ & 347 & 191 & 346 & 884 \\
\hline Mean (SD) & $66.7(15.01)$ & $65.6(14.62)$ & $65.7(14.19)$ & $66.0(14.60)$ \\
\hline Median & 68.1 & 65.8 & 67.2 & 67.1 \\
\hline Min-max & $18-104$ & $21-102$ & $12-98$ & $12-104$ \\
\hline
\end{tabular}

\section{Results}

\section{Demographics and baseline characteristics}

All patients had a history of diabetes for $\geq 10$ years (type 2 $78.4 \%$ overall; type $121.6 \%$ overall), and current diabetic retinopathy. The majority of patients were overweight (body mass index, median $28.7 \mathrm{~kg} / \mathrm{m}^{2}$ overall), male $(68 \%)$, and Caucasian ( $>80 \%$ in all treatment groups and sub-population), and between 51 and 65 years. The demographics and baseline characteristics were mostly similar between the two studies and treatment groups (OCT30, OCT20, and placebo in study 1 , and OCT30 and placebo in study 2). Hypertension was the most common comorbidity in all treatment groups. A summary of the baseline characteristics is presented in Table 2 .

\section{Treatment exposure}

The median duration of exposure to treatment was comparable between the treatment groups: OCT20 = 188 weeks; ОСТ30 $=184$ weeks; and placebo $=$ 204 weeks. Overall, approximately $40 \%$ of the patients in all the three treatment groups had duration of exposure greater than 208 weeks; the maximum duration of exposure to treatment was 300 weeks ( $\sim 5.7$ years $)$.

\section{Safety evaluation}

Overall, the most common AEs reported in the OCT20, OCT30, and placebo groups, respectively were diarrhea (42.9\% [82/191], 58.0\% [202/348], and 21.0\% [73/347]); cholelithiasis $(40.8 \%$ [78/191], $44.3 \%$ [154/348], and 19.6\% [68/347]); hypoglycemia (17.8\% [34/191], 29.6\% [103/348], and 23.6\% [82/347]); nasopharyngitis (18.8\% [36/191], 24.1\% [84/348], and 28.8\% [100/347]); and hypertension (23.0\% [44/ 191], 20.7\% [72/348], and 23.9\% [83/347], Table 3).

Adverse events led to study drug interruption in $12.0 \%$ (23/ 191) patients on OCT20, $13.8 \%$ (48/348) patients on ОСТ30, and $8.1 \%$ (28/347) of patients receiving placebo. Adverse events (suspected to be study drug related) led to discontinuation in $8.9 \%$ (17/191) of patients on OCT $20,16.7 \%$ (58/348) of patients on OCT30, and 2.6\% (9/347) of patients on placebo. Adverse events (not suspected to be study drug related) led to discontinuation in $10.5 \%$ (20/191) of patients on OCT20, 6.9\% (24/348) of patients on ОСТ30, and 8.1\% (28/ 347) of patients on placebo. Neoplasms (benign, malignant and unspecified) were reported in a higher number of patients in the placebo groups in both the studies (study 1: OCT20 $=2.6 \%[5 /$ 191], OCT30 $=1.0 \%$ [2/196], placebo $=4.2 \%$ [8/191]; study $2:$ ОСТ30 $=2.6 \%[4 / 152]$ and placebo $=4.5 \%[7 / 156])$.

The rate for hyperglycemia was comparable between octreotide treatment groups and placebo $(\mathrm{OCT} 20=8.9 \%$ $[17 / 191] ; \quad$ ОСТ $30=9.8 \% \quad[34 / 348] ; \quad$ placebo $=8.6 \%$ 
Table 2 Baseline patient characteristics

\begin{tabular}{|c|c|c|c|c|}
\hline \multirow[t]{2}{*}{ Patient characteristics } & \multicolumn{2}{|l|}{ Long-acting octreotide } & \multirow{2}{*}{$\begin{array}{l}\text { Placebo } \\
N=347\end{array}$} & \multirow{2}{*}{$\begin{array}{l}\text { Total } \\
N=886\end{array}$} \\
\hline & $30 \mathrm{mg} N=348$ & $20 \mathrm{mg} N=191$ & & \\
\hline Age (years), median (min-max) & $56.0(20-77)$ & $56.0(24-70)$ & $57.0(23-75)$ & $57.0(20-77)$ \\
\hline Male, $N(\%)$ Female, $N(\%)$ & $230(66.1) 118(33.9)$ & $142(74.3) 49(25.7)$ & $228(65.7) 119(34.3)$ & $600(67.7) 286(32.3)$ \\
\hline BMI, median $\left(\mathrm{kg} / \mathrm{m}^{2}\right)(\min -\max )$ & $28.7(18-58)$ & $27.8(19-50)$ & $29.1(19-56)$ & $28.7(18-58)$ \\
\hline \multicolumn{5}{|l|}{ Summary of diabetes characteristics } \\
\hline Type $1, N(\%)$ & $80(23.0)$ & $35(18.3)$ & $76(21.9)$ & 191 (21.6) \\
\hline Type $2, N(\%)$ & $268(77.0)$ & $156(81.7)$ & $271(78.1)$ & $695(78.4)$ \\
\hline Duration $\geq 15$ years, $N(\%)$ & $155(44.5)$ & $70(36.6)$ & $161(46.4)$ & $386(43.6)$ \\
\hline HbA1c $(\%)$, median (min-max) & $8.10(4.8-13.6)$ & $8.00(4.0-12.5)$ & $8.50(4.7-15.2)$ & $8.20(4.0-15.2)$ \\
\hline Insulin usage, (yes) $N(\%)$ & $215(61.8)$ & $124(64.9)$ & $219(63.1)$ & $558(63.0)$ \\
\hline $\begin{array}{l}\text { Urinary albumin, median }(\mathrm{g} / \mathrm{L}) \\
(\min -\max )\end{array}$ & $0.0170(0.0047-3.5016)$ & $0.0187(0.0047-2.5230)$ & $0.0353(0.0047-2.5125)$ & $0.0212(0.0047-3.5016)$ \\
\hline \multicolumn{5}{|l|}{ Summary of risk factors } \\
\hline Current smoker, $N(\%)$ & $54(15.5)$ & $46(24.1)$ & $64(18.4)$ & $164(18.5)$ \\
\hline $\begin{array}{l}\text { Systolic blood pressure, median } \\
(\min -\max )\end{array}$ & $140.0(80-220)$ & $145.0(100-200)$ & $140.0(94-211)$ & $140.0(80-220)$ \\
\hline $\begin{array}{l}\text { Diastolic blood pressure, median } \\
\text { (min-max) }\end{array}$ & $80.0(40-110)$ & $80.0(57-105)$ & $80.0(50-114)$ & $80.0(40-114)$ \\
\hline Hypertension, (yes) $N(\%)$ & $221(63.5)$ & $109(57.1)$ & $209(60.2)$ & $539(60.8)$ \\
\hline Dyslipidemia, (yes) $N(\%)$ & $10(2.9)$ & $8(4.2)$ & $9(2.6)$ & $27(3.0)$ \\
\hline
\end{tabular}

Percentage are based on $N=$ total number of subjects in the treatment group

$B M I$ body mass index $=$ weight $/\left(\right.$ height $\left.^{2}\right), H b A l c$ hemoglobin A1c

[30/347]); while the OCT30 group experienced higher rates of hypoglycemia compared with OCT20 and placebo groups $($ OCT20 $=17.8 \%$ [34/191]; ОCT30 $=29.6 \%[103 /$ $348]$; placebo $=23.6 \%$ [82/347]). However, events related to diabetic neuropathy were numerically more frequent in the OCT20 group compared with the OCT30 and placebo groups $($ OCT2 $=5.2 \%$ [10/191]; OCT30 $=2.3 \%$ [8/348]; placebo $=2.6 \%[9 / 347]$ ); while peripheral neuropathy and paresthesia were more frequent in the placebo group compared with the octreotide groups (paresthesia: OCT20 = $2.1 \%$ [4/191]; OCT30 $=1.1 \%$ [4/348]; placebo $=2.9 \%[10 /$ 347]; neuropathy peripheral: OCT20 $=0.5 \% \quad[1 / 191]$; OCT $30=2.3 \% \quad[8 / 348] ; \quad$ placebo $=4.6 \% \quad[16 / 347])$. Hypercholesterolemia was less prevalent with octreotide than with placebo $(\mathrm{OCT} 20=2.6 \%[5 / 191]$; OCT $30=5.2 \%$ $[18 / 348]$; placebo $=13.3 \%$ [46/347]). The mean standard deviation [SD] change in weight $(\mathrm{kg})$ from baseline to last value on study was higher in the placebo group vs. the octreotide treatment groups $($ OCT2 $2=-0.66$ [7.179]; ОСТ30 $=0.24$ [7.151]; placebo $=2.43[6.773]$ ).

\section{Safety topics of special interest}

The incidence of cardiac, renal, and hepatic AEs, by SMQ, is summarized in Table 4, and RR estimates are presented as forest plots in Fig. 1.

\section{Cardiac safety}

Adverse events related to cardiac arrhythmias or QT prolongations were comparable across treatment groups (Table 4). For the ECG findings at EOS, mean heart rate declined over the treatment period; however, PR interval increased and a marginal increase was also observed in the duration of QRS complex (heart rate: OCT20 $=-3.4 \mathrm{bpm}$; OCT30 $=-4.4$ bpm; placebo $=-3.3 \mathrm{bpm} ; \mathrm{PR}$ interval: OCT2 $0=6.1 \mathrm{~ms} ;$ OCT30 $=6.9 \mathrm{~ms}$; placebo $=9.0 \mathrm{~ms} ; \mathrm{QRS}$ duration: OCT $20=3.0 \mathrm{~ms} ; \quad$ OCT $30=2.9 \mathrm{~ms} ;$ placebo $=$ $1.7 \mathrm{~ms})$. The changes in Fridericia's corrected QT interval $(\mathrm{QTcF})$ over time were similar across treatment groups when compared to the placebo arm: OCT $20=7.6 \mathrm{~ms}$; OCT $30=7.1 \mathrm{~ms}$; placebo $=4.2 \mathrm{~ms}$. At the EOS assessment, the QTcF outliers did not exceed $480 \mathrm{~ms}$ or $500 \mathrm{~ms}$ in any of the treatment groups. The incidences of categorical changes of QTcF were as follows: $+30 \mathrm{~ms}$ : OCT2 $2=13.3$; OCT $30=8.1$; placebo $=11.6$, and for $+60 \mathrm{~ms}$ : OCT $20=$ 1.7 ; ОСТ $30=1.5$; placebo $=0.6$.

Events potentially related to Torsades de pointes (fall, syncope, cardiac arrest, and loss of consciousness) were similar between the OCT30 and placebo treatment groups. In total, three instances of sudden death occurred during the trial period: OCT $20=$ no cases; OCT $30=2$ cases; placebo $=1$ case. All three patients (aged 51, 52, and 63 years) had 
Table 3 Most frequent adverse events by preferred term, $\geq 10 \%$ in any long-acting octreotide treatment group

\begin{tabular}{|c|c|c|c|}
\hline \multirow[t]{2}{*}{ Adverse events } & \multicolumn{2}{|c|}{ Long-acting octreotide } & \multirow{2}{*}{$\begin{array}{l}\text { Placebo } \\
N=347 \\
n(\%)\end{array}$} \\
\hline & $\begin{array}{l}30 \mathrm{mg} \\
N=348 \\
n(\%)\end{array}$ & $\begin{array}{l}20 \mathrm{mg} \\
N=191 \\
n(\%)\end{array}$ & \\
\hline $\begin{array}{l}\text { Total patients with an } \\
\mathrm{AE}\end{array}$ & $341(98.0)$ & $189(99.0)$ & $337(97.1)$ \\
\hline Diarrhea & $202(58.0)$ & $82(42.9)$ & $73(21.0)$ \\
\hline Cholelithiasis & $154(44.3)$ & $78(40.8)$ & $68(19.6)$ \\
\hline Hypoglycemia & $103(29.6)$ & $34(17.8)$ & $82(23.6)$ \\
\hline Nasopharyngitis & $84(24.1)$ & $36(18.8)$ & $100(28.8)$ \\
\hline Hypertension & $72(20.7)$ & $44(23.0)$ & $83(23.9)$ \\
\hline Influenza & $52(14.9)$ & $28(14.7)$ & $70(20.2)$ \\
\hline Nausea & $56(16.1)$ & $17(8.9)$ & $53(15.3)$ \\
\hline Macular edema & $46(13.2)$ & $21(11.0)$ & $43(12.4)$ \\
\hline Abdominal pain & $51(14.7)$ & $16(8.4)$ & $29(8.4)$ \\
\hline Diabetic nephropathy & $27(7.8)$ & $32(16.8)$ & $32(9.2)$ \\
\hline Back pain & $37(10.6)$ & $20(10.5)$ & $39(11.2)$ \\
\hline Vitreous hemorrhage & $38(10.9)$ & $18(9.4)$ & $62(17.9)$ \\
\hline Pain in extremity & $40(11.5)$ & $16(8.4)$ & $38(11.0)$ \\
\hline Headache & $42(12.1)$ & $12(6.3)$ & $43(12.4)$ \\
\hline Vomiting & $42(12.1)$ & $12(6.3)$ & $40(11.5)$ \\
\hline Cough & $41(11.8)$ & $11(5.8)$ & $40(11.5)$ \\
\hline Cataract & $26(7.5)$ & $24(12.6)$ & $36(10.4)$ \\
\hline Dizziness & $38(10.9)$ & $11(5.8)$ & $35(10.1)$ \\
\hline Anemia & $44(12.6)$ & $5(2.6)$ & $34(9.8)$ \\
\hline Flatulence & $43(12.4)$ & $6(3.1)$ & $12(3.5)$ \\
\hline Urinary tract infection & $36(10.3)$ & $12(6.3)$ & $33(9.5)$ \\
\hline
\end{tabular}

pre-existing hypertension in addition to diabetes and died at home while sleeping. No autopsy reports were available. The investigators assessed the deaths as not related to study treatment.

The overall incidence of AEs related to cardiac ischemia (SMQ, Table 4) was lower in the octreotide treatment arms compared with the placebo arm (OCT20 $=12.6 \%$ [24/191]; OCT30 $=10.6 \%$ [37/348]; placebo $=15.3 \%[53 / 347])$. The incidence of events related to coronary artery disease (CAD) and angina pectoris (AP) were also lower in the treatment arms compared with the placebo arm (CAD: OCT20 $=1.6 \%[3 / 191]$, OCT30 $=3.4 \%[12 / 348]$, placebo $=5.8 \%$ [20/347]; AP: OCT20 $=2.6 \%$ [5/191], ОСТ $30=$ $1.4 \%[5 / 348]$, placebo $=4.3 \%[15 / 347])$. The incidence of events related to myocardial infarction was however, similar between OCT30 and placebo groups and comparatively higher in the OCT20 group (OCT20 $=5.2 \%$ [10/191]; OCT30 $=2.9 \%[10 / 348] ;$ placebo $=2.9 \%[10 / 347])$.

Based on the individual preferred terms, the incidences of left ventricular hypertrophy (OCT20 $=1.6 \%$ [3/191]; OCT30 $=1.1 \% \quad[4 / 348] ;$ placebo $=3.2 \% \quad[11 / 347])$ and
Table 4 Hepatic, cardiac, and renal adverse events by treatment group and sub-populations defined by baseline eGFR

\begin{tabular}{|c|c|c|c|}
\hline \multirow[t]{2}{*}{ Adverse events } & \multicolumn{2}{|c|}{ Long-acting octreotide } & \multirow{2}{*}{$\begin{array}{l}\text { Placebo } \\
n(\%)\end{array}$} \\
\hline & $\begin{array}{l}30 \mathrm{mg} \\
n(\%)\end{array}$ & $\begin{array}{l}20 \mathrm{mg} \\
n(\%)\end{array}$ & \\
\hline \multicolumn{4}{|c|}{ Baseline eGFR $\geq 60 \mathrm{~mL} / \mathrm{min} / 1.73 \mathrm{~m}^{2}$} \\
\hline$N$ & 237 & 133 & 238 \\
\hline $\begin{array}{l}\text { Cardiac, QT, and } \\
\text { arrhythmias }\end{array}$ & $28(11.8)$ & $14(10.5)$ & $25(10.5)$ \\
\hline Cardiac, ischemias & $27(11.4)$ & $16(12.0)$ & $30(12.6)$ \\
\hline Liver & $31(13.1)$ & $11(8.3)$ & $14(5.9)$ \\
\hline Renal & $6(2.5)$ & $6(4.5)$ & $11(4.6)$ \\
\hline \multicolumn{4}{|c|}{ Baseline eGFR $<60 \mathrm{~mL} / \mathrm{min} / 1.73 \mathrm{~m}^{2}$} \\
\hline$N$ & 110 & 58 & 108 \\
\hline $\begin{array}{l}\text { Cardiac, QT, and } \\
\text { arrhythmias }\end{array}$ & $10(9.1)$ & $6(10.3)$ & $10(9.3)$ \\
\hline Cardiac, ischemias & $10(9.1)$ & $8(13.8)$ & $23(21.3)$ \\
\hline Liver & $18(16.4)$ & $4(6.9)$ & $10(9.3)$ \\
\hline Renal & $16(14.5)$ & $5(8.6)$ & $14(13.0)$ \\
\hline \multicolumn{4}{|l|}{ All patients } \\
\hline$N$ & 348 & 191 & 347 \\
\hline $\begin{array}{l}\text { Cardiac, QT, and } \\
\text { arrhythmias }\end{array}$ & $38(10.9)$ & $20(10.5)$ & $35(10.1)$ \\
\hline Cardiac, ischemias & $37(10.6)$ & $24(12.6)$ & $53(15.3)$ \\
\hline Liver & $49(14.1)$ & 15 (7.9) & $24(6.9)$ \\
\hline Renal & $22(6.3)$ & $11(5.8)$ & $25(7.2)$ \\
\hline
\end{tabular}

A patient with multiple occurrence of an $\mathrm{AE}$ under a preferred term is counted only once under the preferred term. MedDRA version 18. 0 has been used for the reporting of adverse events. Narrow SMQ search: liver (hepatic failure, fibrosis, and cirrhosis and other liver damage related conditions; hepatitis, non-infectious; liver-related investigations, signs and symptoms; cholestasis and jaundice of hepatic origin). Broad SMQ search: cardiac, QT and arrhythmias (arrhythmia related investigations,signs and symptoms, cardiac arrhythmia terms [including bradyarrhythmias and tachyarrhythmias]); cardiac, ischemias (ischemic heart disease); renal (acute renal failure)

increased blood pressure $(\mathrm{OCT} 20=2.6 \%$ [5/191]; OCT30 $=1.7 \%$ [6/348]; placebo $=4.9 \%[17 / 347])$ were lower with octreotide than for placebo.

\section{Renal safety}

The mean baseline eGFR was evenly distributed across treatment groups (OCT 20, OCT30 and placebo). There was a gradual decline in eGFR over the treatment period and the trend was similar across all the 3 treatment groups. The overall incidences of renal AEs (identified by SMQ search) were comparable between treatment groups $($ OCT20 $=$ $5.8 \%[11 / 191] ;$ ОСТ $30=6.3 \%[22 / 348] ;$ placebo $=7.2 \%$ [25/347]). The most commonly reported event term identified by renal SMQ search was renal failure $(\mathrm{OCT} 20=2.6 \%$ 
Fig. 1 Forest plot of overall relative risk of incidence of hepatic, renal, and cardiac adverse events (sub-populations defined by renal function and all patients). Incidence calculated as number of subjects with any by number of subjects at risk. Mantel-Haenszel estimate adjusted for study as stratification factor is used for $\mathrm{RR}$ and $95 \%$ confidence interva treatment-emergent AE divided

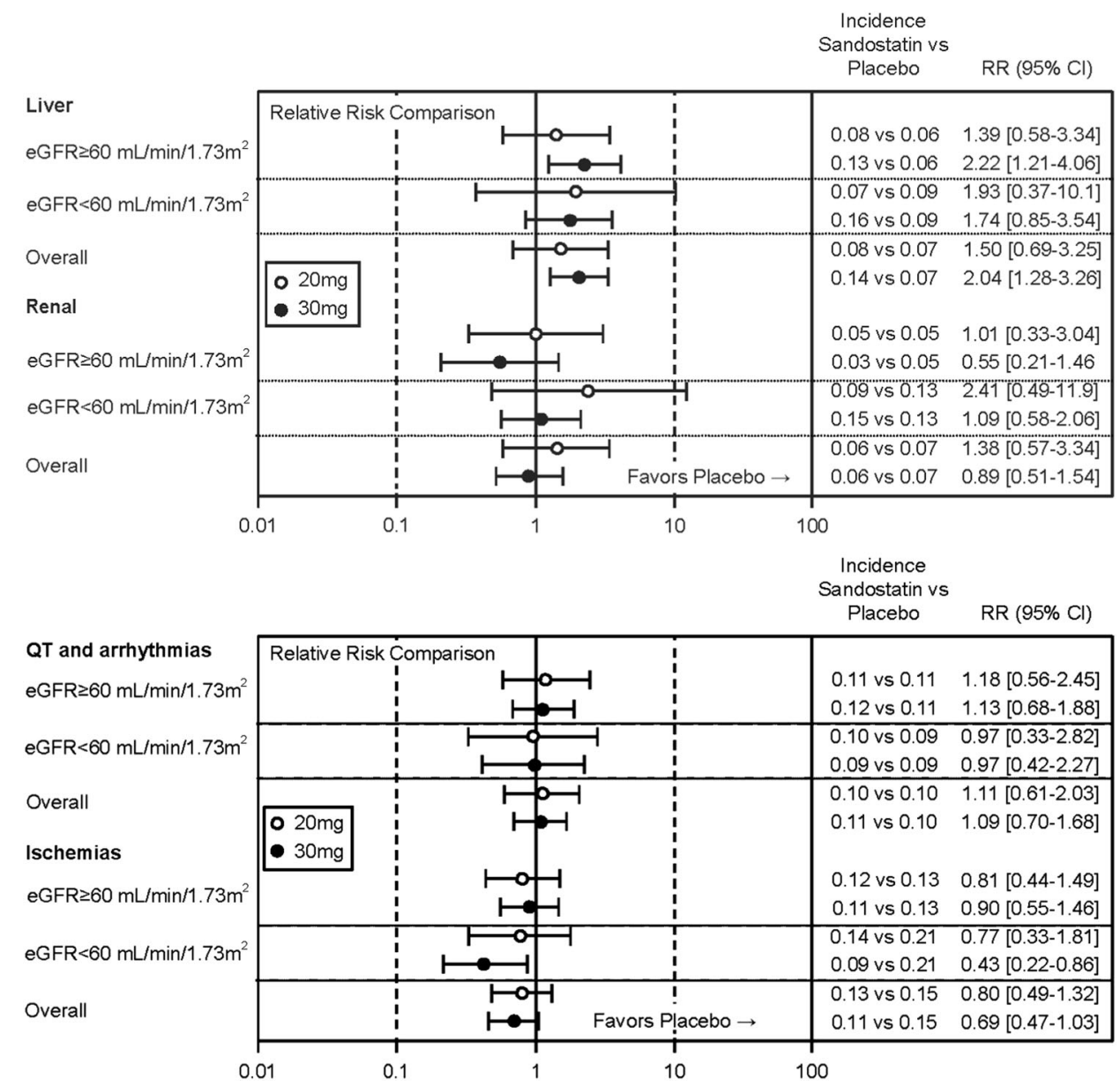

Incidence

Sandostatin vs

$[5 / 191], \quad$ OCT30 $=3.4 \% \quad[12 / 348] ;$ placebo $=3.7 \% \quad[13 /$ 347]).

fatal outcome, cardiac disorder was identified as the most common cause with incidences of $4.2 \%$ (8/191) on OCT20, $2 \%(7 / 348)$ on OCT30 and $2 \%(7 / 347)$ on placebo, respectively.

Overall, the frequency of serious adverse events (SAEs) was similar between treatment groups, but the SAEs suspected to be related to study drug were reported more frequently in the active treatment groups (OCT20 $=7.9 \%$ $[15 / 191] ;$ OCT30 $=10.1 \%$ [35/348]; placebo $=3.5 \%[12 /$ 347]). The most common SAEs suspected to be drug-related ( $>0.5 \%$ of all study patients) were cholelithiasis (OCT20 $=$ $2.6 \%$ [5 of 191], ОСТ30 $=4.3 \%$ [15 of 348], placebo $=$ $0.3 \%$ [1/347]); hypoglycemia (OCT20 $=2.1 \%$ [4/191], OCT30 $=1.7 \% \quad[6 / 348], \quad$ placebo $=0 \%) ; \quad$ cholecystitis $($ OCT20 $=0.5 \%$ [1/191], OCT30 $=1.1 \%$ [4/348], placebo $=0.6 \%[2 / 347])$, diarrhea $($ OCT20 $=1.0 \%$ [2/191], OCT30 $=0.6 \%[2 / 348]$, placebo $=0.6 \%[2 / 347])$, abdominal pain $(\mathrm{OCT} 20=0.5 \%$ [1/191], OCT30 $=0.9 \%$ [3/348], placebo $=0.3 \%$ [1/347]), bile duct stone $($ OCT20 $=0.5 \%$ [1/191], ОСТ $30=0.6 \% \quad[2 / 348], \quad$ placebo $=0.6 \% \quad[2 / 347])$, and cholecystitis chronic $(\mathrm{OCT} 20=1.0 \%$ [2/191], ОCT30 $=$ $0.9 \%$ [3/348], placebo $=0 \%$ ). Only one case of cardiac SAE was suspected to be study drug-related (OCT30 group 
$=0.3 \%[1 / 348])$ and the respective incidences of cardiac SAEs that were not suspected to be study drug related were as follows: OCT2 $=9.4 \%(18 / 191)$; ОCT30 $=9.2 \%(32 /$ $348)$; placebo $=14.7 \%(51 / 347)$. Of note, the renal and urinary disorders related SAEs were not suspected to be study drug-related $($ OCT2 $0=7.9 \%$ [15/191]; OCT30 $=$ $3.4 \%$ [12/348]; placebo $=4.3 \%[15 / 347])$.

\section{Discussion}

The safety profile of octreotide is well established [6, 16-21]. However the long-term effects of octreotide on hepatic, cardiac, and renal safety have only been assessed in uncontrolled settings until now. The pooled analysis of data from the long-term safety follow-up of the two clinical trials presented in the current study, allows for a unique safety review of octreotide. The safety data were both placebocontrolled and had a long median duration of treatment of over 3.5 years. Furthermore, the underlying study population of patients with diabetic retinopathy (not an approved indication for octreotide) provided an opportunity to assess the long-term safety of octreotide in a high-risk population which would potentially be at increased risk for adverse outcomes.

Based on the RR analysis, the long-term use of octreotide was not associated with an increased cardiac risk in these patients regardless of the presence of other risk factors. The incidences of cardiac events (QT prolongation and arrhythmia) for the OCT20 and OCT30 groups were comparable to placebo (OCT20, $\mathrm{RR}=1.11 \quad[95 \% \mathrm{CI}$, 0.61-2.03]; OCT30, RR $=1.09$ [95\% CI, 0.70-1.68]). However, the incidences of cardiac ischemic events were lower in the octreotide treated patients and the risk of these events decreased upon octreotide treatment compared with placebo (OCT20, RR $=0.80$ [95\% CI, 0.49-1.32]; ОCT30, $\mathrm{RR}=0.69$ [95\% CI, 0.47-1.03]). It is noteworthy that fewer coronary artery disease events occurred on treatment with octreotide than placebo. No SAEs reported in the SOC of cardiac disorders were suspected to be related to octreotide.

The frequency of liver-related AEs was higher in patients receiving octreotide (ОСТ20 and ОСТ30) than in the placebo group. As one might expect, the overall prevalence of hepatic steatosis in this study was high, as this condition is associated with diabetes [22-24]. In the general population, the prevalence of fatty liver is estimated to be $20 \%$, while it could be as high as $70 \%$ in obese patients with type 2 diabetes mellitus [22]. This event was the most frequent in all groups including the placebo group and appeared to occur more frequently in the octreotide treatment groups, although this observation was noted in only one of the studies (study 1). The incidence of other liver-related events was low and showed no particular clinical concern for severity. Only a few events of transaminase increase were reported as AEs. Liver chemistry elevations were generally mild and transient, and occurred within the first months of therapy. In the majority of these cases, the liver laboratory values returned to normal without discontinuation of octreotide. Transient and slight elevations of liver enzymes are a well-known class effect observed with SSAs. Other hepatic events (observed in patients on octreotide treatment [OCT20 and OCT30]: hepatomegaly, $n=6$; cholestasis, $n=3$; hepatic cirrhosis, $n=2$; ascites, $n=2$; portal hypertensive gastropathy, $n=1$; hepatic encephalopathy, $n$ $=1$; hepatitis, $n=1$; non-alcoholic steatohepatitis, $n=1$ ) occurred at very low numbers, and no clinically relevant imbalance was seen between octreotide and placebo groups.

The overall renal function profile was similar for both studies. The RR of renal events in both the octreotide treatment groups was not significant compared with the placebo treatment group. This was also apparent from the post-baseline change in eGFR values; indicating that the effect of disease on the renal function surpassed any treatment effect. This is consistent with data from the literature indicating that octreotide has no detrimental effect on renal hemodynamics and tubular function [25-27].

In summary, the results from these unique long-term placebo-controlled studies confirm the established safety profile of long-acting octreotide in patients with diabetic retinopathy, and also in particular demonstrate the low risk of hepatic, cardiac, and renal toxicity.

Acknowledgements We thank the patients and the investigators for their participation in the study; research nurses, trial coordinators, and operations staff for their contributions. We would like to thank the Independent Data Monitoring Committee members Frederick Ferris MD, Gary Koch PhD, Stanley Chang MD, and Lawrence Phillips MD for their contribution to the study. We thank Archana Rai and Anamika Gulati (Novartis Healthcare Private Limited, Hyderabad, India) for providing medical editorial assistance with this article.

Funding The study was funded by Novartis Pharmaceuticals Corporation.

Author Contributions P.J., M.P., S.S., A.L., G.H., and J.E. contributed to the design of the pooled safety analysis and data interpretation. M.P. performed statistical analyzes. R.P., G.M., and A.C. with inputs from all authors contributed towards data analysis and data interpretation. All authors critically reviewed the manuscript for important intellectual content and approved the final version of the manuscript.

\section{Compliance with ethical standards}

Conflict of interest G.H., M.P., S.S., A.L., P.J., and J.E. are employees of Novartis. R.P. has been Principal investigator of research studies from Novartis, has received research grants from Novartis, Pfizer, Viropharma and IBSA, has been occasional consultant for 
Novartis, Ipsen, Pfizer, Viropharma, Ferring, and Italfarmaco, has received Fees and Honoraria for presentations from Novartis and Shire. A.C. is a member of the advisory board Novartis Europe and is recipient of unrestricted funds from Ipsen, Novartis, Pfizer, and Serono for research in neuroendocrinology. G.M. declares that she has no competing interests.

Open Access This article is distributed under the terms of the Creative Commons Attribution 4.0 International License (http://crea tivecommons.org/licenses/by/4.0/), which permits unrestricted use, distribution, and reproduction in any medium, provided you give appropriate credit to the original author(s) and the source, provide a link to the Creative Commons license, and indicate if changes were made.

\section{References}

1. P.E. Battershill, S.P. Clissold, Octreotide. A review of its pharmacodynamic and pharmacokinetic properties, and therapeutic potential in conditions associated with excessive peptide secretion. Drugs 38, 658-702 (1989)

2. M.B. Grant, R.N. Mames, C. Fitzgerald, K.M. Hazariwala, R. Cooper-DeHoff et al., The efficacy of octreotide in the therapy of severe nonproliferative and early proliferative diabetic retinopathy: a randomized controlled study. Diabetes Care 23, 504-509 (2000)

3. S.L. Hyer, P.S. Sharp, R.A. Brooks, J.M. Burrin, E.M. Kohner, Continuous subcutaneous octreotide infusion markedly suppresses IGF-I levels whilst only partially suppressing GH secretion in diabetics with retinopathy. Acta Endocrinol. 120, 187-194 (1989)

4. P. Marbach, W. Bauer, D. Bodmer, U. Briner, C. Bruns et al., Discovery and development of somatostatin agonists. Pharm Biotechnol. 11, 183-209 (1998)

5. Y.C. Patel, Somatostatin and its receptor family. Front. Neuroendocrinol. 20, 157-198 (1999)

6. J. Bornschein, I. Drozdov, P. Malfertheiner, Octreotide LAR: safety and tolerability issues. Expert Opin. Drug Saf. 8, 755-768 (2009)

7. A. Giustina, I. Karamouzis, I. Patelli, G. Mazziotti, Octreotide for acromegaly treatment: a reappraisal. Expert Opin. Pharmacother. 14, 2433-2447 (2013)

8. W.H. Ludlam, L. Anthony, Safety review: dose optimization of somatostatin analogs in patients with acromegaly and neuroendocrine tumors. Adv. Ther. 28, 825-841 (2011)

9. A. Colao, R.S. Auriemma, M. Galdiero, P. Cappabianca, L.M. Cavallo et al., Impact of somatostatin analogs versus surgery on glucose metabolism in acromegaly: results of a 5-year observational, open, prospective study. J. Clin. Endocrinol. Metab. 94, 528-537 (2009)

10. G. Mazziotti, T. Porcelli, F. Bogazzi, G. Bugari, S. Cannavo et al., Effects of high-dose octreotide LAR on glucose metabolism in patients with acromegaly inadequately controlled by conventional somatostatin analog therapy. Eur. J. Endocrinol. 164, 341-347 (2011)

11. G. Mazziotti, I. Floriani, S. Bonadonna, V. Torri, P. Chanson, A. Giustina, Effects of somatostatin analogs on glucose homeostasis: a metaanalysis of acromegaly studies. J. Clin. Endocrinol. Metab. 94, 1500-1508 (2009)
12. L.M. Fatti, M. Scacchi, E. Lavezzi, F. Pecori Giraldi, M. De Martin et al., Effects of treatment with somatostatin analogues on QT interval duration in acromegalic patients. Clin. Endocrinol. 65, 626-630 (2006)

13. A. Colao, R.S. Auriemma, M. Galdiero, G. Lombardi, R. Pivonello, Effects of initial therapy for five years with somatostatin analogs for acromegaly on growth hormone and insulin-like growth factor-I levels, tumor shrinkage, and cardiovascular disease: a prospective study. J. Clin. Endocrinol. Metab. 94, 3746-3756 (2009)

14. L. Warszawski, L. Kasuki, R. Sa, C.M. Dos Santos Silva, I. Volschan et al., Low frequency of cardiac arrhythmias and lack of structural heart disease in medically-naive acromegaly patients: a prospective study at baseline and after 1 year of somatostatin analogs treatment. Pituitary 19, 582-589 (2016)

15. L.A. Stevens, J. Coresh, T. Greene, A.S. Levey, Assessing kidney function-measured and estimated glomerular filtration rate. N. Engl. J. Med. 354, 2473-2483 (2006)

16. Administration USFaD. Octreotide. http://www.accessdata.fda. gov/drugsatfda_docs/label/2003/19667scm044_Sandostatin_lbl. pdf. Accessed 30 Mar 2016

17. L. Anthony, P.U. Freda, From somatostatin to octreotide LAR: evolution of a somatostatin analog. Curr. Med. Res. Opin. 25, 2989-2999 (2009)

18. L. Katznelson, J.L. Atkinson, D.M. Cook, S.Z. Ezzat, A.H Hamrahian et al., American Association of Clinical Endocrinologists medical guidelines for clinical practice for the diagnosis and treatment of acromegaly-2011 update. Endocr. Pract. 17(Suppl 4), 1-44 (2011)

19. M. Pavel, M. Kidd, I. Modlin, Systemic therapeutic options for carcinoid. Semin. Oncol. 40, 84-99 (2013)

20. B. Astruc, P. Marbach, H. Bouterfa, C. Denot, M. Safari et al., Long-acting octreotide and prolonged-release lanreotide formulations have different pharmacokinetic profiles. J. Clin. Pharmacol. 45, 836-844 (2005)

21. J.C. Barbare, O. Bouche, F. Bonnetain, L. Dahan, C. LombardBohas et al., Treatment of advanced hepatocellular carcinoma with long-acting octreotide: a phase III multicentre, randomised, double blind placebo-controlled study. Eur. J. Cancer 45, 1788-1797 (2009)

22. H.B. Bhatt, R.J. Smith, Fatty liver disease in diabetes mellitus. Hepatobiliary Surg. Nutr. 4, 101-108 (2015)

23. J.M. Hazlehurst, C. Woods, T. Marjot, J.F. Cobbold, J.W. Tomlinson, Non-alcoholic fatty liver disease and diabetes. Metabolism 65, 1096-1108 (2016)

24. J. Richard, I. Lingvay, Hepatic steatosis and type 2 diabetes: current and future treatment considerations. Expert Rev. Cardiovasc. Ther. 9, 321-328 (2011)

25. A. Caroli, N. Perico, A. Perna, L. Antiga, P. Brambilla et al., Effect of long-acting somatostatin analogue on kidney and cyst growth in autosomal dominant polycystic kidney disease (ALADIN): a randomised, placebo-controlled, multicentre trial. Lancet 382, 1485-1495 (2013)

26. L.H. Ottesen, N.K. Aagaard, M. Kiszka-Kanowitz, M. Rehling, J. $\mathrm{H}$. Henriksen et al., Effects of a long-acting formulation of octreotide on renal function and renal sodium handling in cirrhotic patients with portal hypertension: a randomized, double-blind, controlled trial. Hepatology 34, 471-477 (2001)

27. H. Sun, S. Zou, K.A. Candiotti, Y. Peng, Q. Zhang et al., Octreotide attenuates acute kidney injury after hepatic ischemia and reperfusion by enhancing autophagy. Sci. Rep. 7, 1-9 (2017) 